separate dorsal and ventral halves, the dorsal axons are still able to find their way despite the absence of channels to guide them. Another possible cue is the nearby neurones, which in whole embryos appear to act as 'stepping stones' or 'guideposts'. Laser destruction of these guideposts leads to disoriented growth of specific axons (Raper, J.A., Bastiani, M.J. and Goodman, C.S. J. Neurosci. 4, 2329; 1984). In the present experiments, the removal of the proximal guidepost neurone did not prevent the axons of more distal cells from moving initally in the right direction.

In both these experimental systems, molecular dissection could be contemplated, but one must remember that cells in culture are not in their natural environment. Some of the important cues may not be expressed, whereas others may not be relevant in vivo. The finer intricacies of connections depend on the interaction of axons with their continuously changing environment, so that the ultimate level of observation must be the intact, living animal. Recent advances in techniques for intracellular dye injection coupled with video-image enhancement have been harnessed brilliantly by Purves and Hadley on page $\mathbf{4 0 4}$ of this issue. The superior cervical ganglion in the neck of the rat is an accessible cluster of cells nicely suited to this technique; by using image intensification, the cell can be photographed without obviously harming it. They first expose the ganglion, fill a particular cell with fluorescent dye and photograph its branching pattern. Several days or even a month later they are able to find the same cell again and repeat the performance. They observe that the branches of the neurone change their shapes progressively over time: nerve cells are not static but are continually adapting, even in the adult animal.

It is precisely in these types of developmental systems that molecular techniques need to be applied. Increasingly, we will identify the appearance of cell surface and extracellular matrix constituents by the use of monoclonal antibodies and discover unique, regionally-localized transcription by in situ hybridization. Such results must be correlated with the kind of developmentally interesting events described in these papers. For example, the appearance of cell-adhesion molecules provides a powerful tool to study development both at the level of cells in the organism and at the level of regulation of gene expression in the cell (Edelman, G.M. Proc. natn. Acad. Sci. U.S.A. 81, 1460; 1984).

It is becoming increasingly apparent that no single factor is responsible for the development of specific neural connections. In the intact animal, there seems to be a redundancy of guidance cues. These papers show that the intact organism must not be forgotten in the quest for molecular answers to developmental questions.

Dennis Summerbell and $R$. Victoria Stirling are at the National Institute for Medical Research, Mill Hill, London NW7 IAA, UK.
Vision

\section{Disparities in depth perception}

\author{
from C. Longuet-Higgins
}

ONE might be surprised that 150 years after the invention of the stereoscope anything remains to be discovered about stereopsis, but on page $\mathbf{4 0 2}$ of this issue Graeme Mitchison and Suzanne McKee describe a novel effect called 'interpolation', which they observe when looking at a particular kind of stereogram. In the past few years stereopsis has had an injection of new interest from two developments in particular: the demonstration by $B$. Julesz and J. Chang. (Biol. Cybernet. 33, $107 ; 1976)$ that pattern perception is not a prerequisite for stereoscopic fusion; and the so-called 'computational paradigm' - the recognition that vision is the outcome of an elaborate neuronal computation for which our eyes supply only the input.

The foremost exponent of the computational paradigm was the late David Marr, whose book, Vision (Freeman, San Francisco, 1982), dealt in some detail with stereopsis. With Tomaso Poggio he produced, in succession, two alternative theories for the mechanism of stereopsis. Though the details of the process are still largely obscure, few visual psychologists would deny Marr's premise that an important preliminary to stereoscopic fusion is the matching of corresponding elements in the two retinal images - the well known 'correspondence problem' of stereopsis. Once the elements have been matched, the visual system can assess the disparities between their retinal positions and draw conclusions about the relative distances of the visible features of the scene.

The observations of Mitchison and McKee bear most directly on the question of how the visual system carries out the matching process. Most of their stereograms, presented for a time too short for the observer to scan the display, were interpreted exactly as predicted by standard stereoscopic theory. The apparent difference in depth between a pair of dots in the stereogram was simply proportional to their horizontal disparity, that is, the difference between their horizontal distances apart in the left and right retinal images. This presupposes that the elements of the two images have been successfully matched; otherwise their disparities are ill-defined.

\section{Erratum}

Due to an editorial mistake in the article by A.R. Gardner-Medwin 'Potential challenge from glia' (Nature 16 May, page 181), it was implied that several long-established properties of glial cells were new findings reported in recent papers. The lines "including . . . . fascination" should be removed from the penultimate sentence of the first paragraph; instead they should complete paragraph 2 , which will then read ". . biological cells, high permeability ..."
With the stereograms that gave predictable impressions of depth, the only uncertainty in the outcome arose from alternative ways of matching the images; the choice between such alternatives seems to be broadly consonant with what is known about the 'disparity gradients' associated with matched pairs of visible elements (Burt, P. and Julesz, B. Science 208, 615; 1980). But the stereograms that Mitchison and McKee find most interesting are those containing regular grids of very closely spaced points, the critical spacing being about $6^{\prime}$ of arc - roughly the angle subtended by adjacent grooves of a nail file held in the hand. At this spacing the visual system seems to 'lose count' of the total number of points in each image, and to estimate the position of the grid by the horizontal disparity between its two edges, ignoring any differences in the numbers of intervening points in the two images; the whole grid is seen as roughly coplanar.

Mitchison and McKee point out that their observations cast doubt on Marr and Poggio's concept of a 'coarse-to-fine vergence strategy', in which the correspondence problem is solved by progressively finer adjustments in the directions of gaze of the two eyes. It will be interesting to see whether they can develop a computational theory of the matching process with a predictive power equal to that of the theories they challenge.

C. Longuet-Higgins is in the Laboratory of Experimental Psychology, University of Sussex, Brighton BNI 9QG, UK.
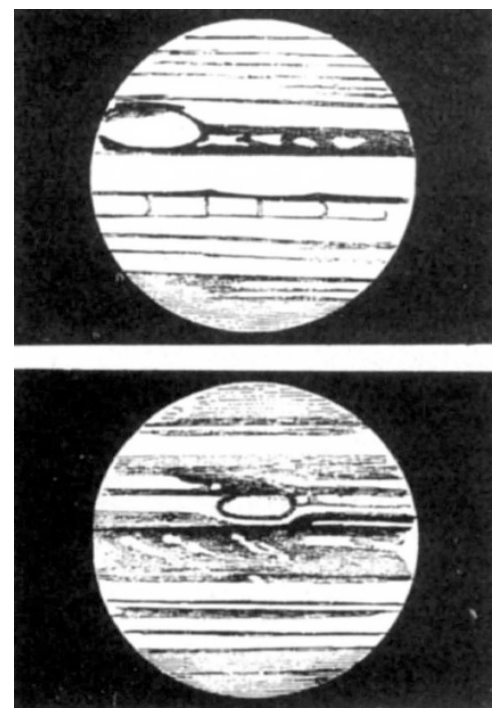

Probably recurrent markings on Jupiter. Top, 27 November 1857 (Dawes); bottom, 12 h 50 min on 25 February 1885 (Denning).

From Nature 32 33, 14 May 1885. 